Administrator, Physics Department, Brookhaven National Laboratory, Upton, NY 11973, USA. For a list of PRT members and their addresses, contact Professor R. A. Young, PRT Chairman for the HRPND, School of Physics, Georgia Institute of Technology, Atlanta, GA 30332, USA.

\section{New Commercial Products}

Announcements of new commercial products are published by the Journal of Applied Crystallography free of charge. The descriptions, up to 300 words or the equivalent if a figure is included, should give the price and the manufacturer's full address. Full or partial inclusion is subject to the Editor's approval and to the space available. All correspondence should be sent to the Editor, Dr A. M. Glazer, Editor Journal of Applied Crystallography, Clarendon Laboratory, University of Oxford, Parks Road, Oxford OX1 3PU, England.

The International Union of Crystallography can assume no responsibility for the accuracy of the claims made. A copy of the version sent to the printer is sent to the compny concerned.

\section{J. Appl. Cryst. (1994). 27, 440}

\section{Mirror Optics}

The Molecular Structure Corporation (MSC) has introduced a high-performance focusing mirror system that produces a three- to fourfold improvement in flux relative to a standard graphite monochromator. The system is completely integrated with the Rigaku R-AXIS imaging-plate system and rotating-anode source. It is easily coupled with the R-AXIS $2 \theta$ stage developed by MSC. It is also sold as a stand-alone unit. Diffraction experiments requiring a high-flux fine-focus $\mathrm{X}$-ray source will benefit from this device.

The double-focusing mirror optics assembly is enclosed in an He-filled chamber with leaded glass front for safe viewing during alignment. The optics consist of a Pt-plated $160 \mathrm{~mm}$ focusing mirror. Both mirrors have a remote control for translation, bending and slit insertion. A fluorescent screen and attenuators are also enclosed in the $\mathrm{He}$-filled chamber, which can be changed by a remote selection switch. The collimator is attached to a high-precision $x y$ assembly.

The system was designed to be easy to align, generally taking less than $30 \mathrm{~min}$. The alignment is unique in that it starts with the mirror nearest the X-ray source and proceeds component by component all the way to the alignment of the $\varphi$ axis.

Molecular Structure Corporation, 3200 Research Forest Drive, The Woodlands, TX 77381, USA.
J. Appl. Cryst. (1994). 27, 440

\section{Efficient SHG/THG Generation of Ultrafast Lasers}

An all-reflective second- and thirdharmonic-generation system is now available from INRAD for use with ultrafast Ti:sapphire lasers. Model 5-050 features a compact reflective design to minimize pulse distortion due to chromatic aberration and group-velocity dispersion that typically are found in lens-based systems. A precision optical delay, polarization-altering optics and crystals are all contained in one compact package. A selection of crystals and optics is available to maintain either fs or ps pulse durations with efficient SHG from 350 to $450 \mathrm{~nm}$ and THG from 233 to $300 \mathrm{~nm}$. Typically, $100 \mathrm{~mW}$ of average THG power can be obtained while maintaining a THG pulse duration of $200 \mathrm{fs}$, as shown by cross-correlation measurements of the third harmonic and fundamental with an INRAD model 5-14BX computerized autocorrelator.

INRAD, 181 Legrand Avenue, Northvale, NJ 07647, USA.

\section{J. Appl. Cryst. (1994). 27, 440}

\section{Cold Probe}

The new FC55 immersion cooler generates temperatures down to $-55^{\circ} \mathrm{C}$ using non-CFC refrigerant gas in a reliable mechanical refrigeration system. The FC55 can be used in place of dry ice or liquid nitrogen as a cooling source for liquid baths or as a cold trapping surface to trap solvents or other condensates from vapor streams. The FC55 generates cooling power of more than $230 \mathrm{BTU} \mathrm{h}^{-1}$ at $-40^{\circ} \mathrm{C}$ and has a footprint of only 10.5 in wide $\times 16.5$ in deep.

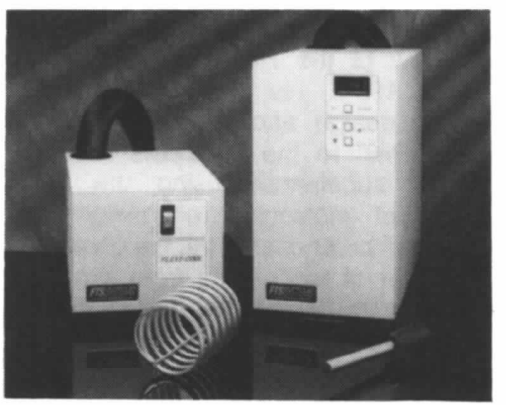

The FC55 cold probe

FTS Systems, Inc., PO Box 158, Rt 209, Stone Ridge, NY 12484, USA.

\section{Book Reviews}

Works intended for notice in this column should be sent direct to the Book-Review Editor (R. F. Bryan, Department of Chemistry, University of Virginia, McCormick Road, Charlottesville, Virginia 22901, USA). As far as practicable, books will be reviewed in a country different from that of publication.

J. Appl. Cryst. (1994). 27, 440-441

The Rietveld method. (IUCr Monograph on Crystallography, No. 5.) Edited by $R$. A. Young. Pp. $\mathrm{x}+$ 298. Oxford: International Union of Crystallography/Oxford University Press, 1993. Price £45.00. ISBN 0-19-855577-6.

In 1969, H. M. Rietveld published his seminal paper on structure refinement based on the complete powder diffraction profile rather than a limited amount of low-angle integrated intensity data. This paper laid the foundation for a dramatic renaissance of powder diffraction, widely regarded at the time as a valuable tool for phase identification and qualitative analysis but of little use for quantitative structure determination. The coming-of-age of Rietveld refinement, as it is now known, was celebrated in June 1989 at an International Workshop on the Rietveld Method, hosted by the Netherlands Energy Research Foundation at $\mathrm{Ri}$ etveld's home institution in Petten and organized by the Commission on Powder Diffraction of the International Union of Crystallography.

The Rietveld method is an outcome of the Petten meeting but is certainly not a Proceedings in the accepted sense. The contributing authors, all of them leaders in the field, were requested to write articles suitable for a book aimed primarily at those with some experience of the technique but also providing some introductory material for beginners. The first drafts of these chapters underwent considerable revision under the guidance and encouragement of the editor and the end result is not only an authoritative and coherent text but also an excellent reference work covering the literature up to the start of 1991 or thereabouts.

The first chapter (R. A. Young) provides an excellent introduction to the Rietveld method. It contains an account of the basic mathematical procedures used in the fitting to the diffraction pattern, the types of parameters to be refined, a list of the peak-shape functions commonly used, corrections for preferred orientation (one of the more troublesome systematic errors afflicting $\mathrm{X}$-ray powder data), some of the popular computer programs currently available, criteria of fit and precision and accuracy, 
and a very helpful section on refinement strategy.

Following a brief but interesting historical account in Chapter 2, by H. M. Rietveld, of the development and acceptance of the method that now bears his name, some of the mathematical aspects of Rietveld refinement are summarized in Chapter 3 (E. Prince), including the method of least squares and its application to the Rietveld model, weights, constrained models, refinement procedures and estimates of uncertainty. In Chapter 4, T.M. Sabine considers processes that modify the flow of radiation in a polycrystalline material and derives simple expressions for absorption, multiple scattering and extinction that can be readily incorporated into the Rietveld model in the form of two refinable parameters: the effective specimen size and the size of the mosaic blocks. Chapter 5 (R. J. Hill) is the longest in the book and gives a comprehensive and pragmatic account of data-collection strategies for Rietveld refinement. Among the many important topics covered are the relative merits of laboratory X-ray, synchrotron X-ray and neutron diffractometers for different types of experiment, the choice of wavelength and resolution, the basic requirements of pattern analysis, how the choices of step increment and counting statistics affect the precision and accuracy of the refinement, and the application of Rietveld refinement to the quantitative analysis of multiphase materials. Background modelling in Rietveld analysis is addressed in Chapter 6, by J. W. Richardson Jr, who describes how a Fourier filtering technique can be used to correct time-of-flight neutron data with broad oscillations in the background caused by the presence of a noncrystalline component in the sample. Some procedures for analytical fitting of laboratory $X$-ray peak profiles in the application of Rietveld analysis are discussed in Chapter 7 by R. L. Snyder. The convolution of sample, spectral and instrumental contributions to the observed profile can be satisfactorily modelled by a split Pearson-VII function. Chapter 8 (R. Delhelz et al.) contains a detailed account of the effect of crystal imperfections on the shape and breadth of the peak profiles in a powder diffraction pattern and of the ways in which information about size and strain can be extracted by Rietveld refinement or pattern decomposition. In Chapter 9, P. Suortti shows how the instrumental profile function can be calculated from the known scattering geometry by ray-tracing or phase-space analysis techniques and also how the background contribution can be divided into an incoherent part that can be calculated explicitly and a coherent part that can be represented by a radial correlation function incorporating the salient features of the thermal and disorder diffuse scattering. In Chapter 10, C. Baerlocher points out how soft constraints, or restraints, can be used to improve the quality of the refinement of complex structures such as zeolites. These restraints, implemented in the form of approximate geometrical relationships in the leastsquares minimization process, are now incorporated into several widely used programs. Chapter 11 (W. I. F. David and J. D. Jorgensen) reviews Rietveld refinement with time-of-flight neutron powder data. The power of this technique with a high-resolution diffractometer, such as the HRPD at the ISIS pulsed neutron source, is strikingly illustrated by examples of anisotropic line broadening in $\mathrm{LaNbO}_{4}$ and a high-precision refinement of the structure of benzene. There are also advantages when special sample environments such as high-pressure cells and furnaces are required. In Chapter 12, R. B. Von Dreele (a co-author with A. C. Larson of the very versatile and widely used GSAS program) describes the extension of Rietveld refinement to a combination of X-ray and neutron powder diffraction data and illustrates how multiple data sets of this type may be the only way to determine the distribution of different atomic species among a number of crystallographic sites. F. Izumi, the author of another very versatile Rietveld refinement program (RIETAN), widely used in Japan, describes some of its features in Chapter 13, including the refinement of incommensurate structures from powder diffraction data and the use of multiple data sets of different types, as exemplified by a refinement of the modulated structure of the high- $T_{C}$ superconductor $\mathrm{Bi}_{2}\left(\mathrm{Sr}_{1-x} \mathrm{Ca}_{x}\right)_{3} \mathrm{Cu}_{2} \mathrm{O}_{8+z}$. Chapter $14(\mathrm{H}$. Toraya) gives an account of patterndecomposition methods, which employ many of the features of Rietveld refinement but do not invoke a structural model. These methods are particularly useful in the initial stages of data analysis of materials about which little is known, and may allow the unit-cell parameters to be determined along with enough individual integrated intensities for $a b$ initio structure solution. The fact that a structural model is not required may also be advantageous in microstructural analysis of size and strain parameters. In the concluding chapter, A. K. Cheetham covers the rapidly developing methodology for the $a b$ initio solution of crystal structures from powder data, an exciting and challenging new area that owes much to the success of the Rietveld technique. He points out that the task of determining the unit-cell parameters and extracting enough integrated intensity data for structure solution by traditional methods is greatly facilitated by the high resolution available at a synchrotron source, whereas neutron data are likely to be most useful in the subsequent Rietveld-refinement stage for the precise determination of atom coordinates. However, the effect of the microstructural characteristics of the sample on the peak shapes will need very careful consideration if pattern decomposition and Rietveld techniques are to be utilized optimally for the determination of very complex structures.

In a book like this, it is inevitable that there is some unevenness in the length and degree of detail in the chapters and that some important topics receive inadequate attention. I would like to see, for example, a more exhaustive discussion of specimen preparation and diffraction geometry, accuracy and significance of the results, the tendency towards empiricism in modelling the peak shapes and the use of symmetryadapted spherical harmonics to correct for preferred orientation and anisotropic line broadening. However, these are not serious criticisms and I recommend this book as a necessity for any diffraction library or for the personal collection of anyone with a serious interest in the application of the Rietveld technique.

DAVID E. COX

Physics Department

Brookhaven National Laboratory

Upton

NY 11973

USA

J. Appl. Cryst. (1994). 27, 441-442

X-ray diffraction at elevated temperatures: a method for in situ process analysis. By $D$. $D$. L. Chung, P. W. DeHaven, $H$. Arnold and $D$. Ghosh. Pp. viii +268 . Weinheim: VCH Verlagsgesellschaft $\mathrm{mbH}$, 1993. Price DM 158. ISBN 0-89573-745-0.

Books of this type, which bring together materials relating to a specialized technique, can be very helpful to new users of a method. The primary literature may be widely dispersed, with pertinent information appearing only as a minor section of a research paper. In many cases, a diligent search is required to find these references and a cursory electronic perusal may 